\title{
CARACTERIZAÇÃO DA DEGRADAÇÃO DE UMA ROCHA UTILIZANDO O DWT
}

\author{
T. F. M. B. DUQUE ${ }^{1}$ e C. L. SCHNEIDER \\ Centro de Tecnologia Mineral - CETEM \\ tduque@cetem.gov.br ${ }^{1}$
}

Artigo submetido em novembro/2013 e aceito em junho/2014

DOI: http://dx.doi.org/10.15628/holos.2014.1841

\section{RESUMO}

Neste trabalho apresenta-se uma análise de um modelo para a caracterização do ensaio DWT completo, para a amostra de rejeito de jigagem de carvão de Criciúma, onde os valores de $\boldsymbol{t}_{n}$ possam ser completamente substituídos por um parâmetro, $\gamma$, que descreve a distribuição granulométrica gerada nos ensaios para todos os tamanhos, além dos parâmetros $\boldsymbol{A}$ e $\boldsymbol{b}$. Além disso, foram realizados dois ensaios padrões, um em Março e outro em Outubro de 2012, a fim de verificar a ocorrência de degradação com o tempo das amostras. O ensaio DWT mede a distribuição granulométrica gerada pelo impacto, em vários níveis de energia, de amostras de partículas com tamanhos definidos. Os resultados são subsequentemente reduzidos a dois parâmetros, $\boldsymbol{A}$ e $\boldsymbol{b}$, característicos do material. O valor do produto $\boldsymbol{A} \times \boldsymbol{b}$ tem sido muito utilizado como um índice de tenacidade de rochas. Verificou-se que a amostra utilizada apresenta valor de $\boldsymbol{A} \times \boldsymbol{b}$ entre 80 e 90 , sendo caracterizada como uma rocha friável, e que ocorreu degradação da amostra com o tempo, em virtude da variação do valor de $\boldsymbol{A} \times \boldsymbol{b}$ para o período estudado. O modelo proposto interpolou satisfatoriamente todas as distribuições geradas nos ensaios com um único valor do parâmetro $\gamma$ para todos os tamanhos testados.

PALAVRAS-CHAVE: Modelamento, Cominuição, Energia, Degradação.

\section{ASSESMENT OF ROCK DEGRADATION USING THE DWT}

\begin{abstract}
This paper presents an analysis of a model for the characterization of the complete DWT test for a sample of coal jigging tailings from Criciúma, where the values of $\boldsymbol{t}_{n}$ can be completely replaced by a single parameter $\gamma$ that describes the particle size distribution generated in the tests for all sizes, besides the parameters $\boldsymbol{A}$ and $\boldsymbol{b}$. In addition, two tests were conducted, one in March and another in October 2012, in order to check the occurrence of degradation with time of the samples. The tests measure the particle size distribution generated by
\end{abstract}

impact at various energy levels using samples of particles with defined sizes. The results are subsequently reduced to two parameters, $\boldsymbol{A}$ and $\boldsymbol{b}$, which are characteristic of the material. The value of the product $\boldsymbol{A} \times \boldsymbol{b}$ has been used as an index of toughness of rocks. It was found that the sample tested has a value of $\boldsymbol{A} \times \boldsymbol{b}$ between 80 and 90, which is defined as a friable rock, and that degradation with time did occur during the period between DWT tests. The proposed model satisfactorily interpolated all distributions generated by the tests with a single parameter for all sizes tested.

KEYWORDS: Modeling, Comminution, Energy, Degradation. 


\section{INTRODUÇÃO}

Recentemente o CETEM adquiriu um equipamento para ensaios de quebra de partículas com energia de impacto controlada. O ensaio mede a distribuição granulométrica gerada pelo impacto, em vários níveis de energia, de amostras de partículas com tamanhos definidos. Os resultados são subsequentemente reduzidos a dois parâmetros, $\boldsymbol{A}$ e $\boldsymbol{b}$, característicos do material (Narayanan e Whiten, 1983). O valor do produto $\boldsymbol{A} \mathbf{x} \boldsymbol{b}$ tem sido muito utilizado como um índice de tenacidade de rochas, sendo que valores $<30$ caracterizam rochas muito tenazes e valores $>127$ caracterizam rochas muito friáveis. Já nas aplicações mais tecnológicas, os valores dos parâmetros $\boldsymbol{A}$ e $\boldsymbol{b}$ são utilizados como parâmetros fundamentais em cálculos metalúrgicos relacionados com operações de britagem de rochas e na moagem em moinhos autógenos e semi-autógenos. A caracterização dos parâmetros de quebra pode ser expandida para fornecer mais detalhes sobre as propriedades dos materiais.

O objetivo deste trabalho é testar um modelo para a caracterização do ensaio DWT onde os valores de $\boldsymbol{t}_{\boldsymbol{n}}$ possam ser completamente substituídos por um parâmetro que descreva a distribuição granulométrica gerada nos ensaios.

\section{METODOLOGIA}

\subsection{Amostras}

Amostras de rejeito de jigagem de carvão de Criciúma foram devidamente preparadas e classificadas em quatro faixas de tamanhos específicos para a realização de um ensaio padrão do DWT. Para cada faixa de tamanho foi escolhido aleatoriamente o número necessário de partículas, a fim de gerar uma ampla e uniforme representação da distribuição das mesmas na amostra inicial. Após essa etapa, para cada intervalo de tamanho as partículas foram separadas em três conjuntos e estes foram, por sua vez, pesados. A Figura 1 ilustra este procedimento. Obteve-se uma média para a massa de cada partícula do conjunto dividindo-se o valor da massa total pelo número de partículas de cada conjunto. Verificou-se também a massa de cada uma das bases, leve e pesada, e dos pesos utilizados nos ensaios.

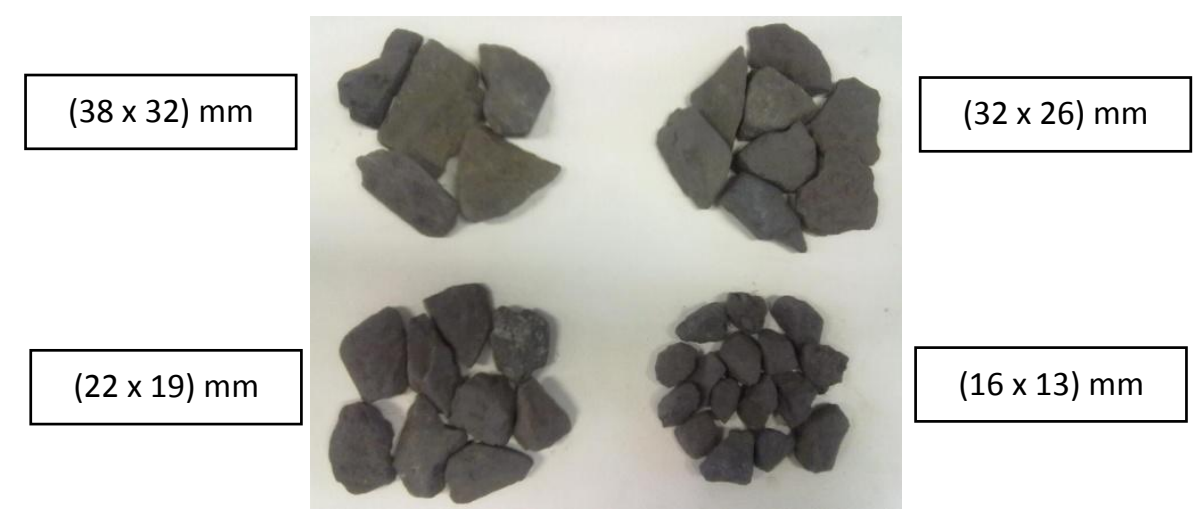

Figura 1 - Amostras de rejeito de jigagem de carvão.

Para cada conjunto foi especificada uma energia, e a partir da combinação dos pesos disponíveis, da energia a ser empregada, da massa de cada partícula e da aceleração da gravidade obteve-se e altura de queda, calculada através da eq.(1): 


$$
H(m)=\frac{\text { Energia }(\mathrm{kWh} / \mathrm{t}) \times 3,6 \times \text { Massa da Partícula }(\mathrm{g})}{g\left(\mathrm{~m} / \mathrm{s}^{2}\right) \times \text { Massa dos pesos }(\mathrm{g})}
$$

Foram realizados dois ensaios padrão, um em Março e outro em Outubro de 2012, a fim de verificar a ocorrência de degradação das amostras com o tempo (Viles, 1997). As condições empregadas nos ensaios podem ser verificadas na Tabela 1.

Tabela 1 - Condições empregadas nos ensaios de DWT.

\begin{tabular}{ccccccccc}
\hline $\begin{array}{c}\text { Faixa de } \\
\text { Tamanho (mm) }\end{array}$ & $\begin{array}{c}\text { Energia } \\
\text { (kWh/t) }\end{array}$ & $\begin{array}{c}\mathbf{N}^{\circ} \text {. de } \\
\text { Partículas }\end{array}$ & \multicolumn{2}{c}{$\begin{array}{c}\text { Massa Total de } \\
\text { Partículas (g) }\end{array}$} & \multicolumn{2}{c}{$\begin{array}{c}\text { Massa por } \\
\text { Partícula (g) }\end{array}$} & \multicolumn{2}{c}{$\begin{array}{c}\text { Altura de Queda } \\
\text { (cm) }\end{array}$} \\
& & & Março & Outubro & Março & Outubro & Março & Outubro \\
$38 \times 32$ & 1,00 & 20 & 873,00 & 985,60 & 43,65 & 49,28 & 64,09 & 72,25 \\
$38 \times 32$ & 0,25 & 20 & 874,40 & 973,10 & 43,72 & 48,66 & 26,75 & 29,70 \\
$38 \times 32$ & 0,10 & 20 & 865,00 & 926,50 & 43,25 & 46,33 & 55,83 & 59,75 \\
$32 \times 26$ & 2,50 & 30 & 1027,20 & 798,70 & 34,24 & 26,62 & 89,77 & 69,73 \\
$32 \times 26$ & 1,00 & 30 & 1025,10 & 797,80 & 34,17 & 26,59 & 83,63 & 64,94 \\
$32 \times 26$ & 0,25 & 30 & 1025,90 & 796,30 & 34,20 & 26,54 & 20,92 & 85,59 \\
$22 \times 19$ & 2,50 & 30 & 397,50 & 362,30 & 13,25 & 12,08 & 81,07 & 73,73 \\
$22 \times 19$ & 1,00 & 30 & 395,90 & 361,90 & 13,20 & 12,06 & 32,30 & 29,46 \\
$22 \times 19$ & 0,25 & 30 & 398,10 & 364,00 & 13,27 & 12,13 & 42,83 & 39,13 \\
$16 \times 13$ & 2,50 & 30 & 148,80 & 141,80 & 4,96 & 4,73 & 30,35 & 28,86 \\
$16 \times 13$ & 1,00 & 30 & 147,00 & 140,60 & 4,90 & 4,69 & 11,99 & 11,45 \\
$16 \times 13$ & 0,25 & 30 & 147,70 & 141,90 & 4,92 & 4,73 & 15,89 & 15,25 \\
\hline
\end{tabular}

\subsection{Ensaios}

A ordem de realização dos ensaios foi determinada da seguinte forma: primeiro todos os conjuntos de partículas que utilizavam a combinação de pesos com a base pesada, depois os conjuntos que utilizavam a combinação de pesos com a base leve. Em ambos os casos, iniciou-se com amostras que só utilizavam as bases e em seguida as que utilizavam as bases e pesos, de forma crescente da massa dos pesos. Os ensaios foram realizados no equipamento JKTech DWT (Figura 2). Esse equipamento é comercializado pelo JKTech da Austrália e se tornou padrão internacional na caracterização das propriedades de quebra de minérios e rochas em geral (Napier-Munn et al., 1996). O ensaio é mundialmente conhecido como DWT (Drop Weight Test). O equipamento consiste de um sistema que deixa cair um peso variável sobre espécimes de rocha de vários tamanhos a partir de diversas alturas.

Para cada ensaio foi ajustada a altura de queda necessária, de forma a atingir o valor mais próximo da altura requerida na Tabela 1, e estas foram anotadas em uma planilha. Após a quebra foi registrada a altura residual para as dez primeiras partículas de cada conjunto e então, procedeuse a quebra das outras partículas. Ao final de cada ensaio, recolheram-se todos os fragmentos das partículas quebradas para que posteriormente fosse feita a análise granulométrica.

As séries de peneira utilizadas sempre continham 15 peneiras com a maior abertura igual ao menor tamanho da faixa, seguindo a ordem decrescente proporcional à $\sqrt{2}$. As partículas com tamanho acima de $19 \mathrm{~mm}$ foram classificadas por peneiramento manual, e as partículas passantes 
em $19 \mathrm{~mm}$ foram classificadas em um peneirador vibratório, durante 20 minutos. As massas retidas em cada fração foram devidamente registradas.

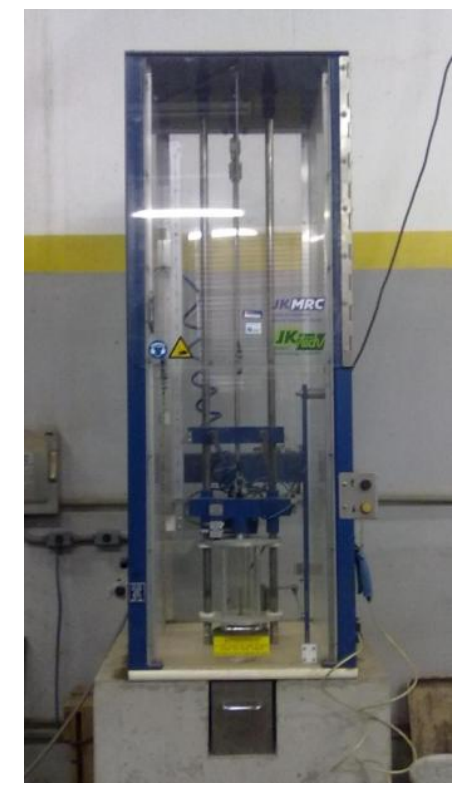

Figura 2 - JKTech DWT instalado no CETEM.

\section{RESULTADOS E DISCUSSÃO}

O modelo proposto descreve a distribuição granulométrica gerada nos produtos de cada tamanho e energia testada. Esse modelo, mais geral, é mostrado na eq. (2) (King, 2012), e resulta da inclusão da equação que descreve $\boldsymbol{t}_{10}$ em função de $\boldsymbol{A}$ e $\boldsymbol{b}$ no modelo da função quebra baseado na distribuição de Rosin-Rammler truncada:

$$
P(D)=1-\left(1-A\left[1-e^{-b E c s}\right]\right)^{\left(\frac{9}{d p}\right)^{\gamma}}
$$

Onde $P(D)$ é a fração cumulativa passante no tamanho de peneira $D$ gerada pela quebra de uma partícula com tamanho representativo $d p$ a partir de uma energia específica de impacto (Ecs). $\boldsymbol{A}, \boldsymbol{b}$ e $\boldsymbol{\gamma}$ são os parâmetros característicos medidos no ensaio. A introdução do parâmetro $\gamma$ dispensa e substitui completamente os diversos parâmetros $\boldsymbol{t}_{\boldsymbol{n}}$ do ensaio DWT.

Na determinação de parâmetros de quebra para aplicação na previsão do desempenho de britadores, por exemplo, os valores dos parâmetros $\boldsymbol{A}$ e $\boldsymbol{b}$, tão somente, não são suficientes. Para este tipo de aplicação, é necessário conhecer a distribuição granulométrica completa que é gerada a partir da quebra de partículas de um dado tamanho com uma dada energia específica. Sabe-se que os valores de $\boldsymbol{A}$ e $\boldsymbol{b}$ variam com o tamanho inicial, mas estas variações podem ser medidas no ensaio completo de DWT. Por outro lado, a distribuição granulométrica completa dos produtos de quebra é caracterizada por uma série de parâmetros derivados das medições, conhecidos como $\boldsymbol{t}_{\boldsymbol{n}}$. Os $t_{n}$ são definidos como a fração de material produzido na quebra que passa na abertura de peneira $\boldsymbol{n}$ vezes menor que o tamanho original. Assim, para valores conhecidos de $\boldsymbol{t}_{\mathbf{2}}, \boldsymbol{t}_{\mathbf{4}}, \boldsymbol{t}_{\mathbf{1 0}}, \boldsymbol{t}_{\mathbf{2 0}} \mathrm{e}$ $\boldsymbol{t}_{50}$, por exemplo, podemos calcular a distribuição granulométrica do produto da quebra de uma partícula de tamanho conhecido (em um britador, por exemplo). É possível tabular os valores de 
$\boldsymbol{t}_{\boldsymbol{n}}$ em função da energia específica de impacto, mas as determinações são ofuscadas por problemas de natureza estatística. Resultados obtidos a partir de diversas rochas de características distintas indicam que os valores de $\boldsymbol{t}_{\boldsymbol{n}}$ seguem um padrão comum, porém esta propriedade nunca foi explorada.

Os resultados dos ensaios completos de DWT realizados com a mesma amostra com defasagem de seis meses são mostrados na Tabela 2 para cada tamanho testado. Os resultados globais de todos os tamanhos analisados simultaneamente, como normalmente é feito no ensaio padrão, são mostrados na Tabela 3. Os valores de $\boldsymbol{A}$ e $\boldsymbol{b}$ variam bastante com o tamanho de partícula testado, sendo que os valores médios de $\boldsymbol{A}$ e $\boldsymbol{b}$ foram 44,72 e 1,82, para o mês de Março; e 45,37 e 1,99 para o mês de Outubro, respectivamente (linha preta nos gráficos da Figura 3). Esta variação com o tamanho de partícula é comumente observada na maioria dos materiais testados.

Tabela 2 - Resultados da análise individual dos ensaios completos de DWT.

\begin{tabular}{ccccccccc}
\hline $\begin{array}{c}\text { Faixa de } \\
\begin{array}{c}\text { Tamanho } \\
\text { (mm) }\end{array}\end{array}$ & \multicolumn{2}{c}{$\boldsymbol{A}$} & \multicolumn{2}{c}{$\boldsymbol{b}$} & \multicolumn{2}{c}{$\gamma$} & \multicolumn{2}{c}{ Axb } \\
& Março & Outubro & Março & Outubro & Março & Outubro & Março & Outubro \\
$38 \times 32$ & 63,98349 & 46,75675 & 1,38139 & 2,35532 & 0,78225 & 0,78646 & 88,38587 & 110,12695 \\
$32 \times 26$ & 52,37974 & 52,51187 & 1,67914 & 2,07092 & 0,72088 & 0,60821 & 87,95278 & 108,74813 \\
$22 \times 19$ & 52,60103 & 53,16350 & 1,50992 & 1,40913 & 0,77211 & 0,75468 & 79,42321 & 74,91421 \\
$16 \times 13$ & 38,86302 & 39,75450 & 1,53308 & 2,52353 & 0,74825 & 0,73339 & 59,58001 & 100,32157 \\
\hline
\end{tabular}
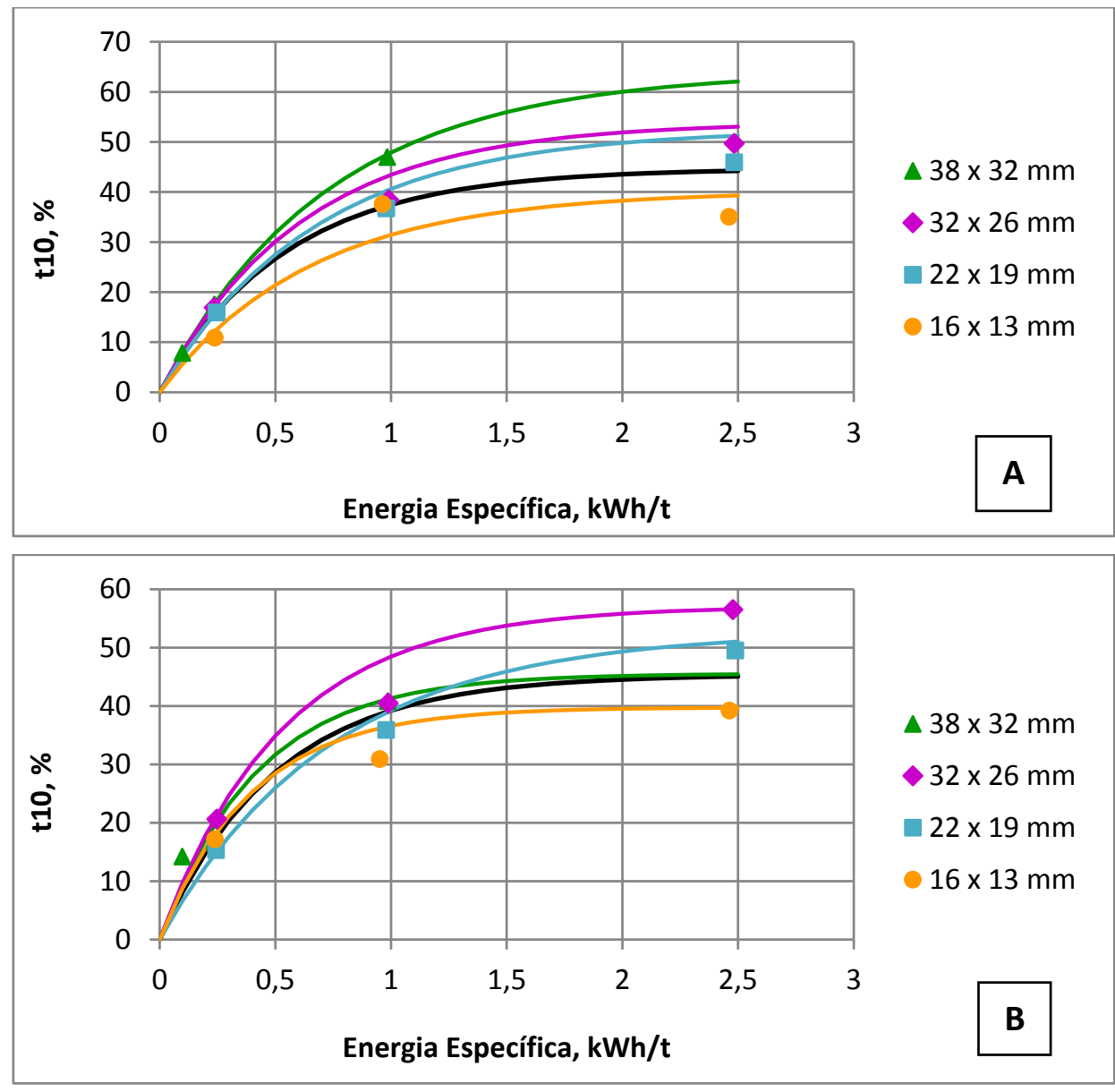

Figura 3 - Resultados dos ensaios completos de DWT, para Março (A) e Outubro (B). 
Tabela 3 - Resultados da análise total dos ensaios completos de DWT.

\begin{tabular}{ccccc}
\hline Ensaio & $\boldsymbol{A}$ & $\boldsymbol{b}$ & $\boldsymbol{\gamma}$ & $\boldsymbol{A x \boldsymbol { b }}$ \\
Março & 44,71658 & 1,82110 & 0,76383 & 81,43352 \\
Outubro & 45,36731 & 1,99923 & 0,73571 & 90,69961 \\
\hline
\end{tabular}

Tabela 4 - Tabela de classificação.

\begin{tabular}{cccccccc}
\hline Propriedade & $\begin{array}{c}\text { Muito } \\
\text { Tenaz }\end{array}$ & Tenaz & $\begin{array}{c}\text { Moderadamente } \\
\text { Tenaz }\end{array}$ & Médio & $\begin{array}{c}\text { Moderadamente } \\
\text { Friável }\end{array}$ & Friável & $\begin{array}{c}\text { Muito } \\
\text { Friável }\end{array}$ \\
$\boldsymbol{A x b}$ & $<30$ & $30-38$ & $38-43$ & $43-56$ & $56-67$ & $67-127$ & $>127$ \\
\hline
\end{tabular}

Fonte - http://www.jktech.com.au/sites/default/files/brochures/LabServices_DWTest_Indetail.pdf

A Tabela 4 contém uma classificação qualitativa da tenacidade ou friabilidade dos materiais em termos dos valores de $\boldsymbol{A} \boldsymbol{x} \boldsymbol{b}$ característicos. Pela tabela acima pode-se classificar o rejeito de jigagem de carvão como uma rocha friável (Axb: 67-127). Para os diversos tamanhos os valores de $\boldsymbol{A}$ variam entre 39 e 64 enquanto os valores de $\boldsymbol{b}$ variam entre 1,38 e 1,69, para o mês de Março; e no mês de Outubro os valores variam entre 40 e 47 para $\boldsymbol{A}$, enquanto para $\boldsymbol{b}$ variam entre 1,41 e 2,52 .

Enquanto foi determinado que os valores de $\boldsymbol{A}$ e $\boldsymbol{b}$ variam significativamente de tamanho para tamanho, o ensaio demonstrou que um único valor do parâmetro $\gamma$ interpola todas as distribuições granulométricas medidas nos produtos da quebra. Distribuições típicas dos dois ensaios são mostradas na Figura 4. Pode-se concluir que o novo modelo descreve bem o ensaio de DWT com a amostra de rejeito de jigagem de carvão. Todos os cálculos podem ser facilmente organizados em uma planilha do tipo Excel ${ }^{\mathrm{TM}}$.
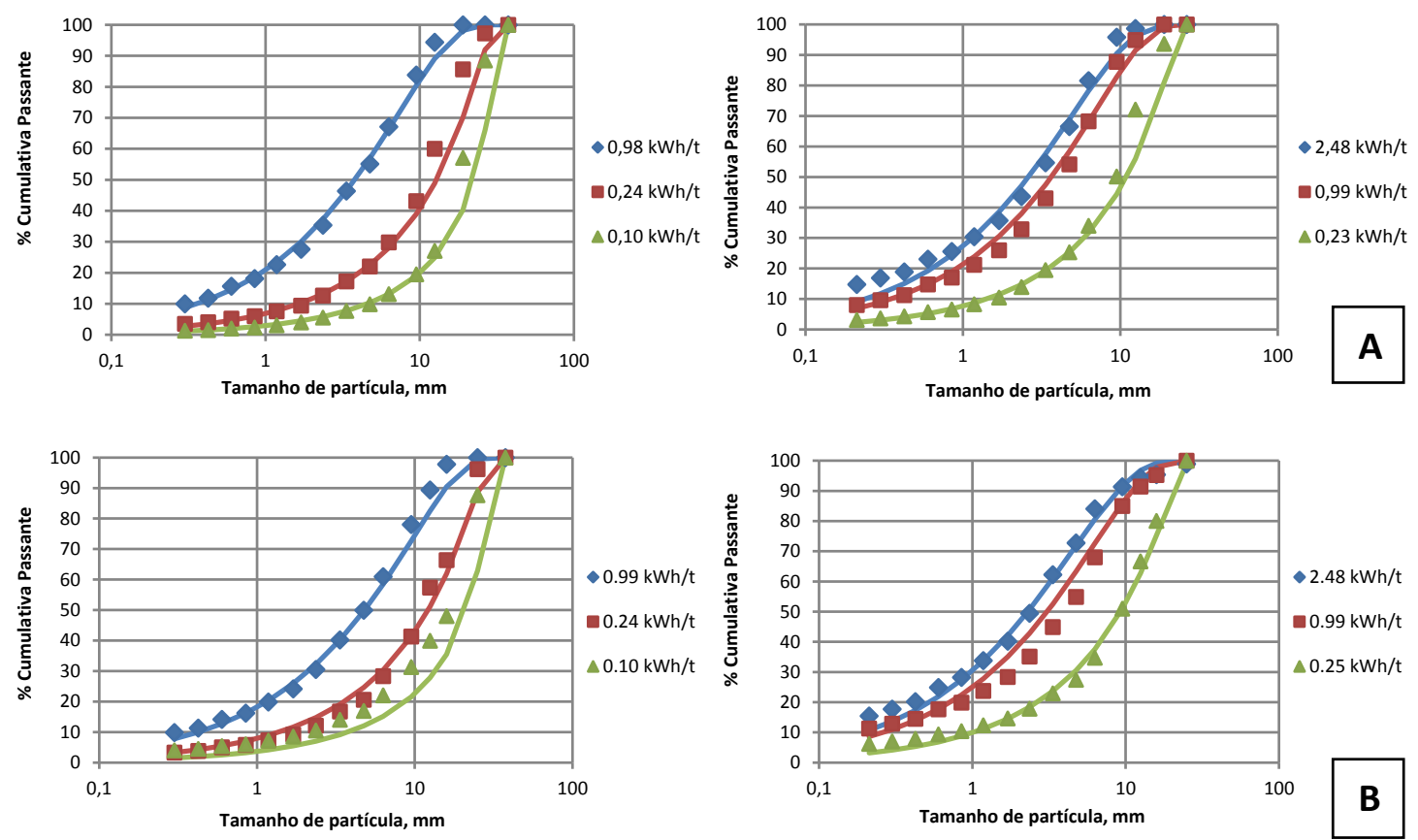

Figura 4 - Distribuições granulométricas geradas na quebra de partículas de 38x32 mm (esquerda) e 32×26 mm (direita), sendo (A) referente ao ensaio realizado em Março, e (B) em Outubro. Pontos representam valores medidos e linhas representam a eq.(2). 
Constatou-se também a ocorrência de degradação das amostras, visto que o valor de $\boldsymbol{A x \boldsymbol { b }}$ sofreu um aumento de aproximadamente $11 \%$ no período relatado, ou seja, as partículas utilizadas no ensaio do mês de Outubro estão mais friáveis com relação às mesmas amostras testadas em Março do mesmo ano. Os resultados também mostram que a degradação se reflete principalmente nos valores do parâmetro $\boldsymbol{b}$, indicando que as amostras degradadas produzem a mesma quantidade de finos com menor energia específica de impacto.

\section{CONCLUSÕES}

Os resultados mostram que existe uma tendência à degradação da amostra de rejeito de jigagem de carvão ao longo do período de sete meses no qual a amostra preparada foi armazenada. Considerando-se que materiais deste tipo são utilizados em campanhas de caracterização de propriedades físicas, recomenda-se que as amostras a serem analisadas sejam utilizadas com a maior brevidade possível.

O rejeito de jigagem de carvão é um material que necessita ser estocado em pilha com controle de drenagem em função da presença de pirita. Nestes casos a degradação é interessante porque possibilita a cominuição com uma demanda energética menor, viabilizando o reprocessamento deste resíduo.

A metodologia utilizada para a análise dos resultados dos ensaios DWT produz resultados compatíveis com a metodologia tradicional e, ao mesmo tempo, incrementa as informações sobre as propriedades de quebra dos materiais testados. Os modelos de britadores, por exemplo, não mais necessitariam de uma tabela de valores de $\boldsymbol{t}_{\boldsymbol{n}}$ em função de $\boldsymbol{t}_{10}$, e o valor do parâmetro $\gamma$ é suficiente para o cálculo dos $\boldsymbol{t}_{n}$.

\section{REFERÊNCIAS BIBLIOGRÁFICAS}

1. NARAYANAN, S.S.; WHITEN W.J, Breakage Characteristics of Ores for Ball Mill Modeling, (1983), Proceedings AUSIMM, No. 286, pp. 31-39.

2. VILES, H.A. Urban air pollution and the deterioration of buildings and monuments. In: The global environment: science, technology and management, ed. BRUNE, D., CHAPMAN, D.V., GRUYNNE, M.D., PACYNA, J.M., 1997. Scandinavian Science Publisher and Weinheim; VCH: Germany. pp. 599-609.

3. NAPIER-MUNN, T.J.; MORRELL S.; MORRISON R.D.; KOJOVIC, T., Mineral Comminution Circuits - Their Operation and Optimization, (1996), JKMRC Monograph Series in Mining and Mineral Processing 2.

4. KING, R.P., Modeling \& Simulation of Mineral Processing Systems, $2^{\text {nd }}$ Edition (2012), Society for Mining, Metallurgy, and Exploration, Inc., Englewood, Colorado. 